\title{
Fast raycasting using a compound deep image for virtual point light range determination
}

\author{
Jesse $\operatorname{Archer}^{1}(\bowtie)$, Geoff Leach ${ }^{1}$, and Pyarelal Knowles ${ }^{1}$ \\ (c) The Author(s) 2019.
}

Abstract The concept of using multiple deep images, under a variety of different names, has been explored as a possible acceleration approach for finding raygeometry intersections. We leverage recent advances in deep image processing from order independent transparency for fast building of a compound deep image $(C D I)$ using a coherent memory format well suited for raycasting. We explore the use of a CDI and raycasting for the problem of determining distance between virtual point lights (VPLs) and geometry for indirect lighting, with the key raycasting step being a small fraction of total frametime.

Keywords deep image; indirect lighting; raycasting

\section{Introduction}

Recently, the use of multiple deep images has been explored as an alternative to a bounding volume hierarchy, the most common acceleration approach for raycasting. Deep images store multiple per-pixel fragments which include color and depth information, compared to $2 \mathrm{D}$ flat images which store only a single color value. Deep images are a view-dependent discretized representation of scene geometry, and a complete, nearly view-independent representation for the purpose of raycasting can be achieved using many deep images from different directions - which we call a compound deep image ( $C D I)$.

Previous approaches have found that building few deep images is fast but slow to raycast due to the need to step between different deep image pixels and directions. Fast raycasting requires many deep images

1 School of Science, RMIT University, Melbourne, 3000, Australia. E-mail: J. Archer, jesse.archer@rmit.edu.au (更); G. Leach, geoff.leach@rmit.edu.au.

Manuscript received: 2019-03-04; accepted: 2019-05-06 from different directions, which allows a ray to be contained in a single pixel with fragments representing geometry intersections. While raycasting such a CDI is fast, previous approaches to building the deep images have been relatively slow and non-realtime, typically using memory incoherent per-pixel linked lists. We present a realtime approach for building a CDI using per-pixel linearised arrays, a memory coherent alternative to linked lists which offers faster raycasting.

This paper explores fast CDI building and raycasting for the problem of range determination using ray-geometry intersections for indirect lighting using virtual point lights (VPLs), as shown in Fig. 1. The primary challenge for realtime rendering of VPLs is determining which VPL applies to what geometry. Recent realtime VPL techniques either assume lights have infinite range and restrict their contribution to geometry using imperfect shadow maps, or limit ranges randomly as unshadowed stochastic VPLs. Restricting the range of each light allows rapid building of a light grid which can be applied to geometry. We show that ranges can be estimated by casting multiple rays per-VPL to nearby geometry. This approach is fast for thousands to millions of VPLs, with realtime raycasting of up to tens of millions of rays.

While range determination has potential to improve visual quality over randomly assigned light ranges, this work omits detailed visual comparisons with other VPL techniques. We instead provide a basic visual comparison with ground-truth path tracing showing that our work gives similar results to single bounce path tracing but with some visual artifacts. The primary focus of this work is improved performance of the CDI building and raycasting steps. We show that, while slower than assigning random 


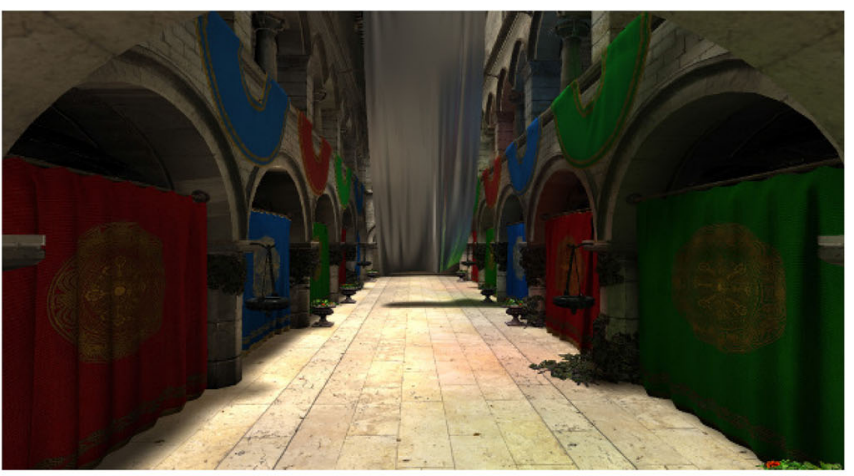

(a) Atrium

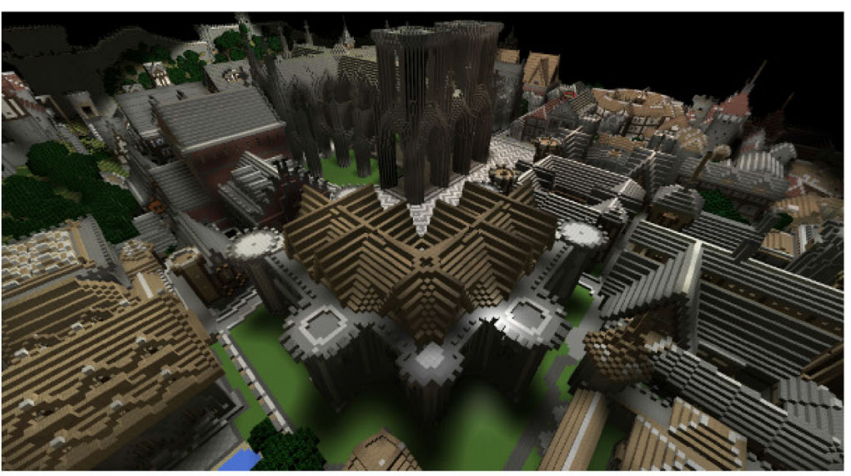

(b) Rungholt

Fig. 1 Indirect lighting using VPLs with a small indoor Atrium scene and large outdoor Rungholt scene.

light sizes, the raycasting step is only approximately $1 \%$ of total frametime.

\section{Related work}

Deep images are primarily used in compositing and transparency, but have recently been applied to various lighting problems. Raycasting using deep images with three deep image directions is presented in Refs. [1, 2] but requires rays to step between pixels. CDIs have been explored for global illumination $[3,4]$ but with non-realtime results, or requiring precomputing which is unsuitable for dynamic scenes. In general, using fewer directions is faster to build while using more directions reduces the need to step between pixels and thus is faster to raycast.

The use of screen-space deep images [5] and unstructured surfel clouds [6] for raycasting has also been presented. However, our focus is raycasting deep images of full object-space scene geometry.

Advances in fast building of deep images in real time have primarily been presented for order independent transparency, requiring both a capture and a sorting stage. Capture using linked lists and linearised arrays has been compared [7, 8]. Linked lists have smaller capture time while arrays are faster to sort and process. Fast sorting of full fragment list data relies on using blocks of GPU registers $[9,10]$ and more recently GPU instruction caching [11].

Indirect lighting is a well known problem; instant radiosity [12] was the first proposed fast VPL technique. Single bounce VPLs are generated from reflective shadow maps (RSMs) [13] which record light bounces from a primary light source. Restricting the contribution of each VPL to nearby visible geometry is achieved in real time either using imperfect shadow maps [14-16], or approximated stochastically by assigning random light ranges or randomly culling unshadowed VPLs for diffuse [17, 18] and glossy lighting [19].

Light grids are a well established approach for rendering scenes with many lights where the range of each light is known and finite. Various approaches for building both 2D [20-22] and 3D [23, 24] grids have been explored. This work uses the hybrid lighting approach [25] for building a 3D grid; linearised arrays were similarly found to offer faster processing of light data than linked lists.

\section{CDI building and raycasting}

\subsection{CDI building}

A compound deep image (CDI) is a collection of deep images from different directions with each deep image stored sequentially using linearised arrays. Many approaches exist for determining unique deep image directions, with previous work sampling using a unit cube from three or more directions, or uniformly distributing directions on a unit sphere. This work samples deep image directions from a hemisphere using spherical coordinates as shown in Fig. 2. Only the top hemisphere is needed as the bottom is indexed by reversing a given ray's direction. Other sampling approaches have previously been used such as recursive zonal equal-area partitioning [4] and uniformly sampling a unit cube [3].

Each pixel in a deep image represents a specific ray in that deep image's direction. The fragment list for the pixel then gives all the geometry intersected by the ray. The ray for a given object-space position is 


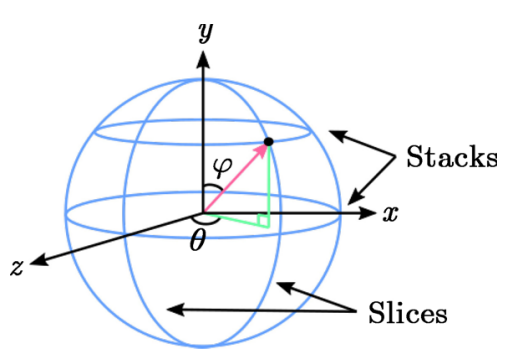

Fig. 2 Direction vector using spherical coordinates.

determined by computing the deep image's screenspace pixel.

A brute force approach for building a CDI rerasterizes geometry for each unique direction as in previous work. However, this approach is expensive for hundreds of directions. We instead use a well known approach for geometry voxelization by rasterizing geometry once and saving fragment data for many directions.

Geometry is rasterized with the camera oriented along the $z$ axis. The normal vector of each triangle is checked in the geometry shader and $x, y, z$ components are swizzled for triangles primarily facing along the $x$ or $y$ axes to ensure each triangle is instead primarily facing along the $z$ axis. Unswizzled object-space positions are passed to the fragment shader. Per-direction orthographic modelviewprojection matrices are iterated and multiplied with the fragment's position, yielding unique per-direction fragments which are added to the appropriate deep images. See Fig. 3.

Many geometry voxelization approaches require conservative rasterization to ensure all geometry is represented. We find that for the problem of VPL

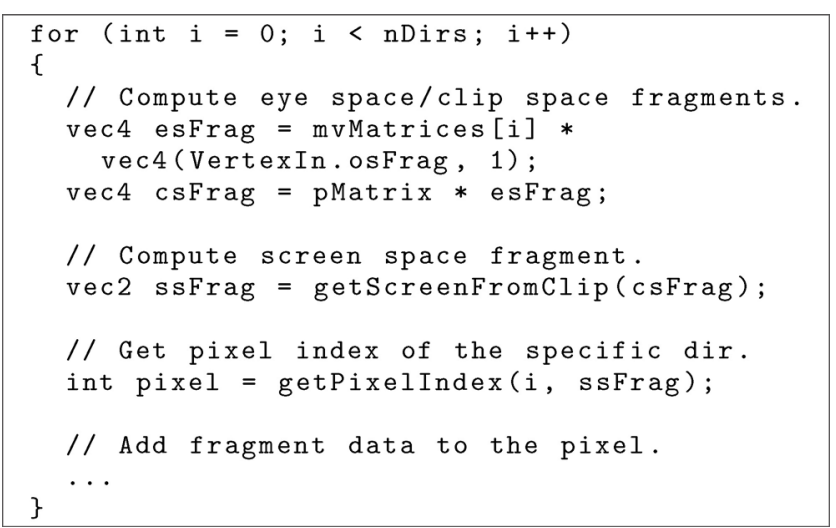

Fig. 3 Computing unique per-direction fragments in the fragment shader from object space positions. range determination, a non-conservative representation suffices, reducing both memory usage and deep image build time. Any missing geometry can cause some VPLs to have larger ranges than needed, which is offset by taking an average length of multiple ray directions.

Sampling geometry primarily facing along the $z$ axis maximises the number of geometry samples generated in the fragment shader. This causes redundant fragment data to be added for deep image directions where geometry is side-on or at an angle where fewer samples are needed, increasing overall CDI memory usage by up to $25 \%$.

Fast raycasting using deep images relies on reading fragment data coherently, so arranging fragment data into appropriate coherent buffers in memory is critical for fast processing on the GPU. Two possible approaches are linked lists and linearised arrays.

Previous deep image raycasting techniques typically used per-pixel linked lists where fragment data can be written in-place as geometry is rasterized. This approach offers fast build time as fragment data is written sequentially during rasterization, but at the expense of slower raycasting, as following the next pointers for each linked list leads to scattered memory reads.

Linearised arrays require two buffers as shown in Fig. 4. Unlike linked lists, in which fragment data can be anywhere, fragment data in linearised arrays for a given pixel is coherent, with all fragments for a given pixel stored contiguously. Linearised arrays are slower to build than linked lists but faster to raycast.

Building a deep image in this format is summarised as follows, based on Ref. [26]:

- Initialise a buffer of per-pixel counts to zero.

- Render geometry and atomically increment counts in the fragment shader.

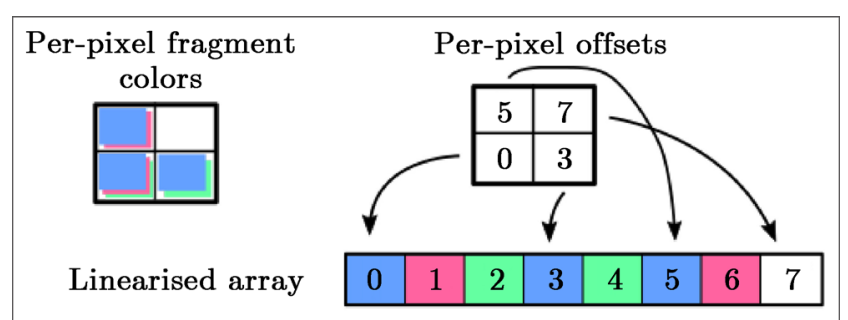

Fig. 4 Per-pixel blue/red/green, blue/green, and blue/red fragment colors stored in linearised arrays. 
- Compute per-pixel offsets from counts using parallel prefix sums.

- Allocate fragment data buffer of size given by final offset.

- Re-render geometry, storing fragments in locations determined by atomically incrementing offsets.

Traversing a pixel's fragment data requires reading the index offset and number of fragments, then reading the fragment data sequentially. Example code is given in Fig. 5 for the three main steps of computing per-pixel counts, adding fragment data to, and traversing the linearised array. The same buffer is used for both fragment counts and offsets. Not shown is computing offsets using a parallel prefix sum scan, which is described in Ref. [27].

A limitation of using linearised arrays is that geometry must be rasterized twice as opposed to linked lists where it is rasterized once. For complex scenes where rasterization is a bottleneck, this can cause capture time for arrays to be up to twice as long as for linked lists. Despite this, linearised arrays have better overall performance, as the CDI is built only once but read many times, and the coherent memory reads of linearised arrays are much faster than the scattered memory reads of linked lists.

Fast raycasting requires each pixel's fragment list to be in depth sorted order. Fast per-pixel fragment sorting depends on maximising use of registers, the fastest available GPU memory. Using registers requires hard coding a sort network with all compareand-swap operations explicitly defined [10]. We use an unrolled bitonic sort network which also provides improved instruction caching [11].

As available per-thread registers are limited, long lists are sorted in blocks. Each block is sorted using a hard-coded sort network followed by a $k$-way merge in local memory of the sorted blocks [9].

Finally, fragment data is written back to global

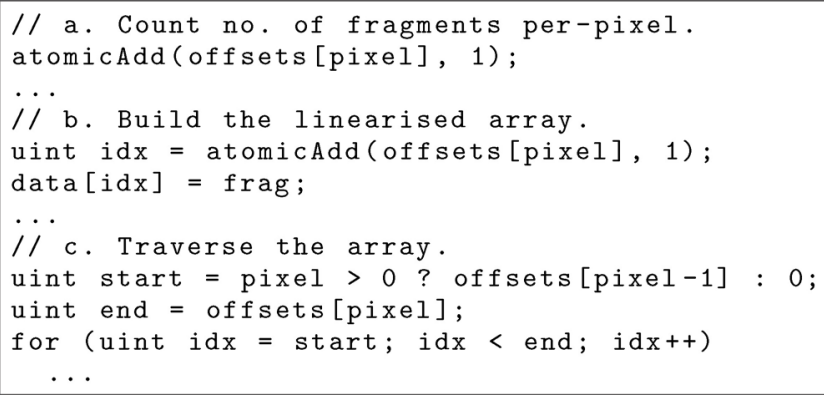

Fig. 5 Adding a fragment to and traversing a pixel's linearised array. memory after sorting. This sort approach is very fast, with reading from and writing back to global memory being up to $90 \%$ of the total sort time. Sorting is thus primarily dominated by memory bandwidth.

An example of part of the final CDI rendered transparently is shown in Fig. 6 where the Atrium scene is shown from many different directions, each represented as a separate deep image. Note that extra fragments are present for some geometry in different directions, leading to overlapping triangles. As geometry is captured from a single rasterization, the same viewport size is used for all deep images, which is large enough to contain the entire scene from any direction.

\subsection{VPL range determination}

We apply a CDI to the problem of range determination of VPLs using broadly the following steps.

- Capture and sort the CDI.

- Render g-buffer from camera's perspective.

- Render RSM from primary light's perspective, giving VPL positions.

- Build buffer of unique VPLs, raycasting using the CDI to determine VPL ranges.

- Build light grid from the VPL buffer.

- Apply the light grid to the g-buffer, giving the final result.

After building the CDI, a global buffer of unique VPLs is built. Scene geometry is rendered from the light's perspective to an RSM, giving VPL positions and colors. To avoid oversampling geometry close to the light, we use a mipmapped RSM and choose positions from higher mipmap levels based on distance to light source. Other approaches for choosing optimal VPL positions from an RSM have been explored but this issue is not the focus of this work.

As VPL positions are calculated, the distance to nearby geometry is determined by raycasting through the previously built CDI. The primary VPL direction is given either by the reflected light direction or the surface normal. Six rays per-VPL are cast, one in the primary direction and five oriented around it.

Each ray indexes the CDI using the closest matching direction. The light's position then determines the pixel matching that specific ray. Distance from the light to geometry is found by stepping through the pixel's fragment list from the beginning until a fragment after the light's position is reached. This linear stepwise search is used as a 


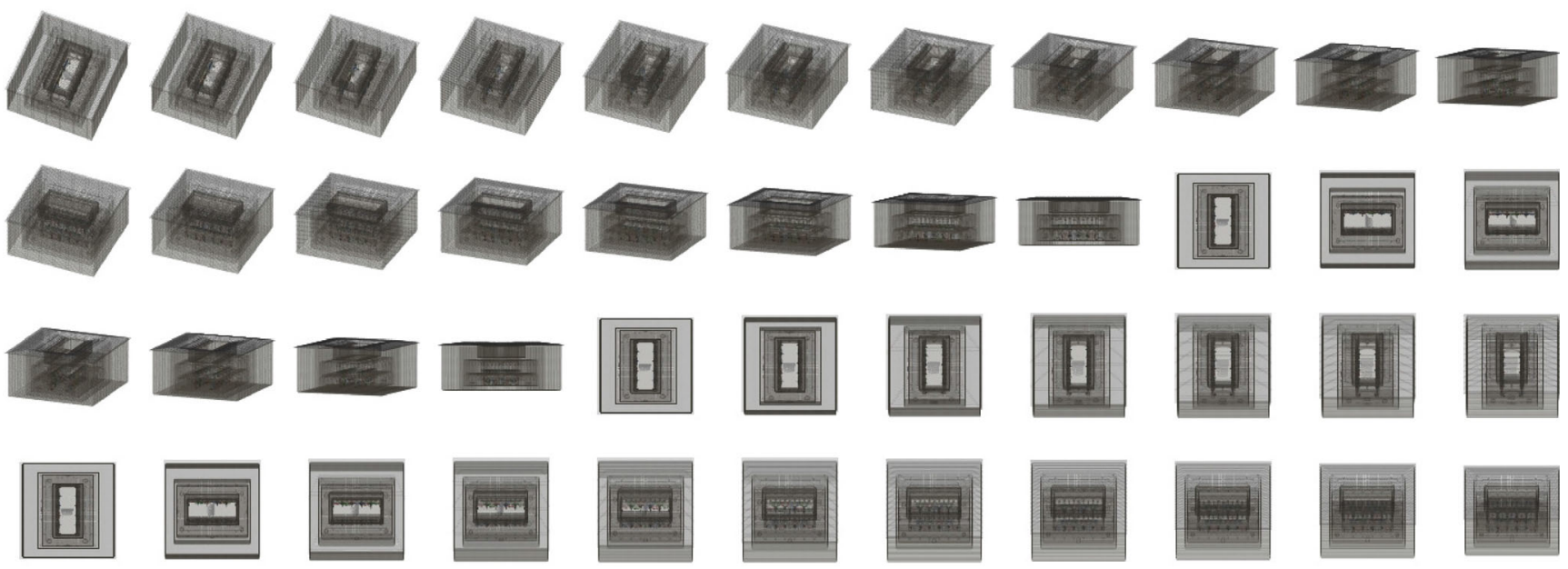

Fig. 6 Part of a CDI showing the Atrium scene as multiple deep images in different directions.

binary search was found to be slower. The difference in depth between the fragment and the light gives an approximate range for the given ray. Fragments are stepped in reverse order for rays in the matching opposite direction. After raycasting, a sphere for the VPL is created with radius of the either the average or maximum distance to geometry.

Scenes typically require many small lights and few large lights. We keep most small lights, discarding those too small to noticeably affect the scene and most large lights. Each non-discarded VPL is atomically added to a global VPL buffer storing position, color, and size.

After building the CDI and determining light sizes we use hybrid lighting [25] as mentioned in Section 2 to build and apply the light grid from the global VPL buffer. 2D light outlines are rasterized at coarse resolution with front and back per-tile depths determined using a line-sphere intersection test. The light's data is then added to all 3D grid cells between the front and back depths for the 2D tile. Light grid data is stored using the same linearised array format as for the deep image, which was similarly found to be faster than using linked lists. A depth mask is built from the g-buffer to avoid adding lights to grid cells that contain no geometry.

As VPLs are not necessarily uniformly distributed in the scene, to prevent some parts of the scene appearing too dim or too bright, per-pixel lighting is adjusted proportionally to the number of applicable VPLs. Lighting is applied using the Cook-Torrance BRDF [28] which is suitable for diffuse and glossy materials.
Other techniques for spawning and applying VPLs with and without light grids have been discussed but are not our primary focus, instead being left for future work. Shadows from the primary light source are calculated separately and not included in performance measurements.

\section{Results}

We compare performance of indirect lighting using VPLs for the two scenes shown in Fig. 1. The first scene is the Sponza Atrium with approximately 280,000 triangles and the second is the Rungholt outdoor town scene with approximately 7 million triangles. These scenes are available from Ref. [29]. The test platform was an NVIDIA GeForce GTX 1060, driver version 390.25. Scenes were rendered at HD $(1920 \times 1080)$ resolution. Time is reported in millisecond.

The primary focus of this work is determining the cost of building and raycasting the CDI for VPL range determination in the context of indirect lighting, with chosen VPLs being added to a light grid. As stated previously, other techniques for choosing and applying VPLs both with and without light grids have been presented and combining range determination with these techniques is left for future work.

Results for the full VPL rendering approach are shown in Table 1 where raycasting is shown in bold and is the fastest VPL rendering step. The CDI uses 169 directions at $64 \times 64$ resolution which represents a hemisphere of $13 \times 13$ stacks and slices. Raycasting is performed when building the global VPL buffer shown 
Table 1 Time for each step of VPL rendering

\begin{tabular}{lrr}
\hline & Atrium & Rungholt \\
\hline Capture deep images & $3.5 \mathrm{~ms}$ & $18.8 \mathrm{~ms}$ \\
Sort deep images & $2.0 \mathrm{~ms}$ & $3.3 \mathrm{~ms}$ \\
Render g-buffer & $1.3 \mathrm{~ms}$ & $8.0 \mathrm{~ms}$ \\
Render RSM & $0.5 \mathrm{~ms}$ & $5.9 \mathrm{~ms}$ \\
Build VPL buffer & $\mathbf{0 . 1 5} \mathrm{ms}$ & $\mathbf{0 . 4} \mathrm{ms}$ \\
Build light grid & $0.7 \mathrm{~ms}$ & $0.5 \mathrm{~ms}$ \\
Apply lighting & $12.0 \mathrm{~ms}$ & $6.1 \mathrm{~ms}$ \\
Total & $20.6 \mathrm{~ms}$ & $43.5 \mathrm{~ms}$ \\
\hline
\end{tabular}

in bold to estimate light sizes and takes approximately $1 \%$ of the total frametime. The RSM is rendered at $256 \times 256$ resolution and contains 65,536 potential VPLs. Only 20,000 VPLs are typically used to avoid oversampling geometry close to the light source, which after raycasting and discarding based on size are reduced to 2000 and 8000 VPLs for the Atrium and Rungholt scenes respectively.

There is little overhead for computing many directions, as shown in Table 2, which compares building the CDI with an increasing number of directions. The Atrium scene is dominated by memory writes but processing remains fast even for more than one hundred directions. There is little difference in performance for the Rungholt scene which is dominated by geometry rasterization being performed twice, once for per-pixel counts and once to write the fragments. For both scenes, total build time scales linearly with the number of directions.

Our CDI approach that builds all directions simultaneously is $20 \times$ to $100 \times$ faster than a brute force approach that builds directions separately. For 169 directions our approach takes 5.5 and $22.1 \mathrm{~ms}$ to build the CDI for the Atrium and Rungholt scenes respectively, while a brute force approach takes 121.3 and $2295.8 \mathrm{~ms}$, with no difference in the final indirect lighting result.

Table 3 shows the cost of raycasting for an increasing number of lights. Time to cast six rays perVPL to estimate size for the Atrium and Rungholt scenes is compared with time to assign a random size

Table 2 CDI build time

\begin{tabular}{ccc}
\hline Directions & Atrium & Rungholt \\
\hline 8 & $1.5 \mathrm{~ms}$ & $18.0 \mathrm{~ms}$ \\
16 & $1.8 \mathrm{~ms}$ & $18.4 \mathrm{~ms}$ \\
32 & $2.0 \mathrm{~ms}$ & $18.6 \mathrm{~ms}$ \\
64 & $3.2 \mathrm{~ms}$ & $19.7 \mathrm{~ms}$ \\
128 & $4.7 \mathrm{~ms}$ & $21.3 \mathrm{~ms}$ \\
\hline
\end{tabular}

Table 3 Raycasting time for Atrium and Rungholt scenes, versus assigning random size VPLs

\begin{tabular}{lrrr}
\hline RSM resolution & $\begin{array}{r}\text { Atrium } \\
\text { raycast }\end{array}$ & $\begin{array}{r}\text { Rungholt } \\
\text { raycast }\end{array}$ & Random \\
\hline $256 \times 256$ & $0.5 \mathrm{~ms}$ & $0.6 \mathrm{~ms}$ & $0.04 \mathrm{~ms}$ \\
$512 \times 512$ & $1.8 \mathrm{~ms}$ & $2.1 \mathrm{~ms}$ & $0.14 \mathrm{~ms}$ \\
$1024 \times 1024$ & $6.0 \mathrm{~ms}$ & $6.9 \mathrm{~ms}$ & $0.5 \mathrm{~ms}$ \\
$2048 \times 2048$ & $20.2 \mathrm{~ms}$ & $23.5 \mathrm{~ms}$ & $2.1 \mathrm{~ms}$ \\
\hline
\end{tabular}

to each light. Lights are spawned for all RSM pixels and none are discarded.

Raycasting time increases nearly linearly with the number of rays, and is approximately $10 \times$ slower than assigning random ranges. While this difference is significant, it remains fast for thousands to millions of VPLs and as shown in Table 1, it is only a small percentage of total frametime. While slower than randomly assigning light ranges, raycasting allows indirect lighting using fewer lights than stochastic approaches where many more lights are required to ensure enough VPLs cover the necessary geometry.

Complexity of geometry has little impact on raycasting performance, with the Rungholt scene being no more than $10 \%$ slower to raycast than the Atrium. While the Rungholt scene has more than $20 \times$ the number of triangles, CDIs for the two scenes are approximately the same size, at $18 \mathrm{MB}$ for 169 directions, which is insignificant compared to the total memory available on most modern GPUs. Fragment list lengths for the Rungholt scene are typically only slightly longer than the Atrium and thus only slightly slower to raycast.

At the highest tested resolution of $2048 \times 2048$, approximately 25 million raycasts are performed in $20 \mathrm{~ms}$, equivalent to raytracing an HD $(1920 \times 1080)$ image with 12 rays per-pixel.

For the Atrium scene, it takes $5.5 \mathrm{~ms}$ to capture and sort the CDI, which compares favourably with other CDI techniques. For the Atrium scene, the deterministically layered depth maps approach [4] takes $373 \mathrm{~ms}$ for both building and computing ambient occlusion, and more than $1000 \mathrm{~ms}$ for computing indirect lighting, although they do not specify how much time is specifically dedicated to building and raycasting. Their results were reported using a GTX780 Ti to render at $800 \times 800$ resolution with 512 deep images of $50 \times 50$ resolution. Older CDI work [3] reports $60 \mathrm{~ms}$ for calculating indirect lighting of the Atrium with a precomputed CDI. 
Multiview and multilayer interactive ray tracing [2] renders three directions rather than many and takes $10 \mathrm{~ms}$ build time for the Atrium using a GTX780 Ti at $720 \times 480$ resolution with deep images rendered at $480 \times 480$ resolution. Again, our build approach is faster given the difference in hardware.

The ground-truth Rungholt outdoor town scene rendered using path tracing with 128 per-pixel samples is shown in Fig. 7, with zero bounce and one bounce respectively. Our approach gives similar results to one bounce path tracing but with visual artifacts where some areas of the scene are darker or brighter than the ground-truth rendering.

Brighter areas are caused by light bleeding from unshadowed VPLs that affect more geometry than necessary while darker areas are caused by parts of the scene being undersampled from the light's perspective in the RSM. Addressing these artifacts requires calculating shadows using imperfect shadow maps and a better sampling approach for spawning VPLs. Range determination of VPLs should improve performance of existing shadow map approaches by reducing the number of maps that need to be

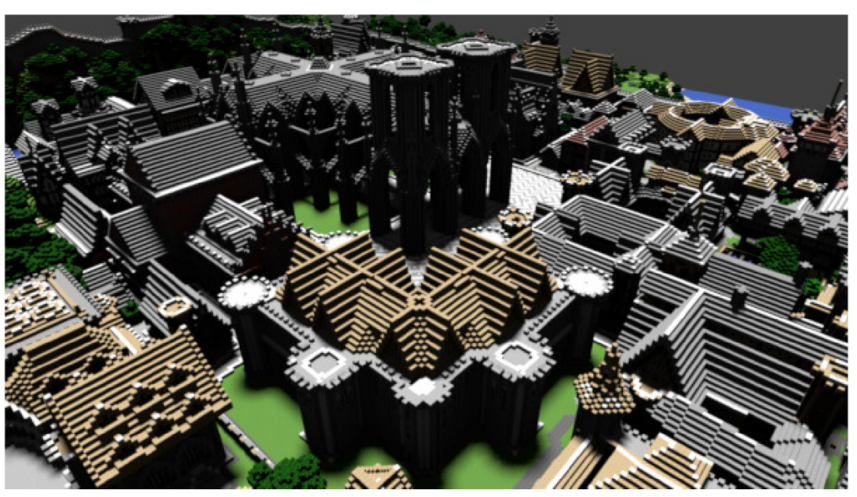

(a) Zero bounce - direct light only

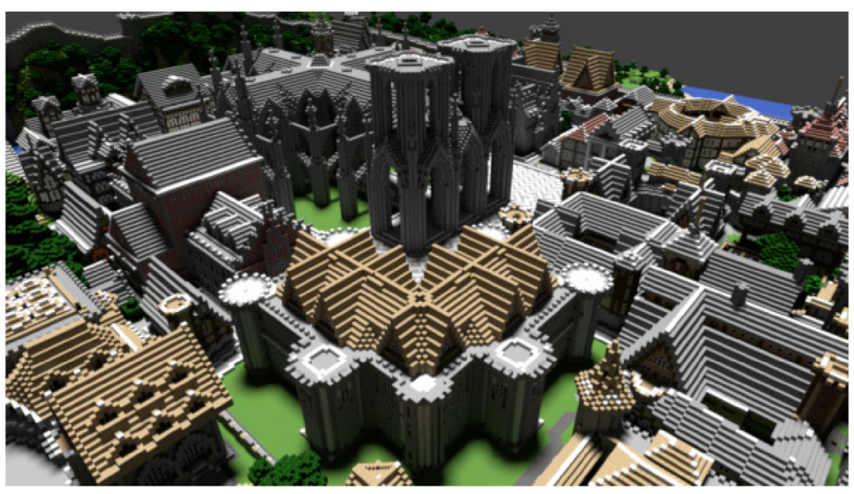

(b) One bounce-direct and indirect light

Fig. 7 Rungholt scene rendered using path tracing with zero and one bounce. tested per-pixel.

\section{Conclusions}

This work has presented a CDI approach that offers fast build time with little memory overhead. The linearised array format used is ideally suited for raycasting due to fragment list memory coherence, even for millions of VPLs.

We use CDIs specifically for building a VPL grid. Previous papers have explored CDIs for ray and path tracing, and applying our coherent CDI structure should similarly improve performance.

Using the CDI to build a light grid shows similar results to one bounce path tracing but with some visual artifacts. Addressing these requires combining imperfect shadow maps and a better sampling approach for spawning VPLs.

Electronic Supplementary Material Supplementary material is available in the online version of this article at https://doi.org/10.1007/s41095-019-0144-1.

\section{References}

[1] Hu, W.; Huang, Y. Y.; Zhang, F.; Yuan, G. D.; Li, W. Ray tracing via GPU rasterization. The Visual Computer Vol. 30, Nos. 6-8, 697-706, 2014.

[2] Vardis, K.; Vasilakis, A. A.; Papaioannou, G. A multiview and multilayer approach for interactive ray tracing. In: Proceedings of the 20th ACM SIGGRAPH Symposium on Interactive 3D Graphics and Games, 171-178, 2016.

[3] Nießner, M.; Schäfer, H.; Stamminger, M. Fast indirect illumination using layered depth images. The Visual Computer Vol. 26, Nos. 6-8, 679-686, 2010.

[4] Aalund, F. P.; Frisvad, J. R.; Bærentzen, J. A. Interactive global illumination effects using deterministically directed layered depth maps. In: Proceedings of the 26th Eurographics Symposium on RenderingExperimental Ideas and Implementations, 2015.

[5] Mara, M.; McGuire, M.; Nowrouzezahrai, D.; Luebke, D. Fast global illumination approximations on deep g-buffers. NVIDIA Technical Report NVR-2014-001. 2014.

[6] Nalbach, O.; Ritschel, T.; Seidel, H.-P. Deep screen space. In: Proceedings of the 18th Meeting of the ACM SIGGRAPH Symposium on Interactive 3D Graphics and Games, 79-86, 2014.

[7] Maule, M.; Comba, J. L. D.; Torchelsen, R.; Bastos, R. Memory-efficient order-independent transparency with dynamic fragment buffer. In: Proceedings of the 
25th SIBGRAPI Conference on Graphics, Patterns and Images, 134-141, 2012.

[8] Knowles, P.; Leach, G.; Zambetta, F. Efficient layered fragment buffer techniques. In: OpenGL Insights. Cozzi, P.; Riccio, C. Eds. CRC Press, 279-292, 2012.

[9] Knowles, P.; Leach, G.; Zambetta, F. Backwards memory allocation and improved OIT. In: Proceedings of Pacific Graphics, Vol. 2013, 59-64, 2013.

[10] Knowles, P.; Leach, G.; Zambetta, F. Fast sorting for exact OIT of complex scenes. The Visual Computer Vol. 30, Nos. 6-8, 603-613, 2014.

[11] Archer, J.; Leach, G. Further improvements to OIT sort performance. In: Proceedings of Computer Graphics International, 147-152, 2018.

[12] Keller, A. Instant radiosity. In: Proceedings of the 24th Annual Conference on Computer Graphics and Interactive Techniques, 49-56, 1997.

[13] Dachsbacher, C.; Stamminger, M. Reflective shadow maps. In: Proceedings of the Symposium on Interactive 3D Graphics and Games, 203-231, 2005.

[14] Ritschel, T.; Grosch, T.; Kim, M. H.; Seidel, H.-P.; Dachsbacher, C.; Kautz, J. Imperfect shadow maps for efficient computation of indirect illumination. ACM Transactions on Graphics Vol. 27, No. 5, 1, 2008.

[15] Ritschel, T.; Eisemann, E.; Ha, I.; Kim, J. D. K.; Seidel, H.-P. Making imperfect shadow maps view-adaptive: High-quality global illumination in large dynamic scenes. Computer Graphics Forum Vol. 30, No. 8, 2258-2269, 2011.

[16] Barák, T.; Bittner, J.; Havran, V. Temporally coherent adaptive sampling for imperfect shadow maps. Computer Graphics Forum Vol. 32, No. 4, 87-96, 2013.

[17] Laurent, G.; Delalandre, C.; de La Rivière, G.; Boubekeur, T. Forward light cuts: A scalable approach to real-time global illumination. Computer Graphics Forum Vol. 35, No. 4, 79-88, 2016.

[18] Tokuyoshi, Y.; Harada, T. Stochastic light culling. Journal of Computer Graphics Techniques Vol. 5, No. 1, 35-60, 2016.

[19] Tokuyoshi, Y.; Harada, T. Stochastic light culling for VPLs on GGX microsurfaces. Computer Graphics Forum Vol. 36, No. 4, 55-63, 2017.

[20] Olsson, O.; Assarsson, U. Tiled shading. Journal of Graphics, GPU, and Game Tools Vol. 15, No. 4, 235251, 2011.

[21] Harada, T.; McKee, J.; Yang, J. C. Forward+: Bringing deferred lighting to the next level. In: Proceedings of the Eurographics-Short Papers, 5-8, 2012.

[22] Bezrati, A. Real-time lighting via light linked list. GPU Pro Vol. 6, 183, 2015.
[23] Olsson, O.; Billeter, M.; Assarsson, U. Clustered deferred and forward shading. In: Proceedings of the 4th ACM SIGGRAPH/Eurographics Conference on HighPerformance Graphics, 87-96, 2012.

[24] Ortegren, K.; Persson, E. Clustered shading: Assigning lights using conservative rasterization in directx 12 . GPU Pro Vol. 7, 43, 2016.

[25] Archer, J.; Leach, G.; Knowles, P.; van Schyndel, R. Hybrid lighting for faster rendering of scenes with many lights. The Visual Computer Vol. 34, Nos. 6-8, 853-862, 2018.

[26] Archer, J.; Leach, G.; van Schyndel, R. GPU based techniques for deep image merging. In: Proceedings of the SIGGRAPH Asia Technical Briefs, Article No. 19, 2017.

[27] Harris, M.; Sengupta, S.; Owens, J. D. Parallel prex sum (scan) with CUDA. GPU Gems Vol. 3, No. 39, 851-876, 2007.

[28] Cook, R. L.; Torrance, K. E. A reflectance model for computer graphics. ACM Transactions on Graphics Vol. 1, No. 1, 7-24, 1982.

[29] McGuire, M. Computer graphics archive. 2017. Available at https://casual-effects.com/data/.

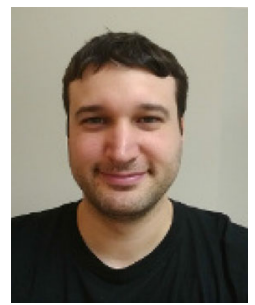

Jesse Archer is a Ph.D. student at RMIT University, Melbourne. His research interests are real-time computer graphics and GPU computing. He completed his Bachelor of Computer Science degree in 2008, Bachelor of IT (Games and Graphics Programming) degree in 2010, and Honours in Computer Science in 2015 at RMIT

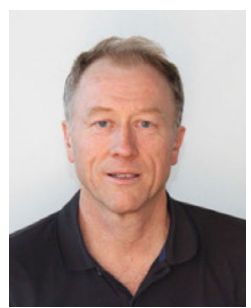

Geoff Leach is a lecturer in the School of Science at RMIT University. His major research interests include computer graphics, computational science, and GPU computing. He teaches mostly computer graphics, and has been using OpenGL since version 1.1. He holds a M.App.Sci. degree from RMIT.

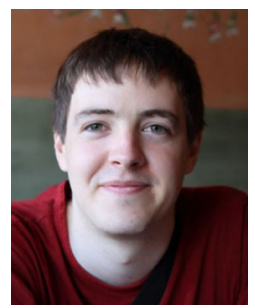

Pyarelal Knowles is a senior software engineer at nVidia. He completed his Ph.D. degree in computer science at RMIT University in 2015, and has research interests in real-time computer graphics and a background in games programming. 
Open Access This article is licensed under a Creative Commons Attribution 4.0 International License, which permits use, sharing, adaptation, distribution and reproduction in any medium or format, as long as you give appropriate credit to the original author(s) and the source, provide a link to the Creative Commons licence, and indicate if changes were made.

The images or other third party material in this article are included in the article's Creative Commons licence, unless indicated otherwise in a credit line to the material. If material is not included in the article's
Creative Commons licence and your intended use is not permitted by statutory regulation or exceeds the permitted use, you will need to obtain permission directly from the copyright holder.

To view a copy of this licence, visit http:// creativecommons.org/licenses/by/4.0/.

Other papers from this open access journal are available free of charge from http://www.springer.com/journal/41095. To submit a manuscript, please go to https://www. editorialmanager.com/cvmj. 